\title{
DETECTION OF NOVEL RESPIRATORY MECHANICS PARAMETERS BY MEANS OF FORCED OSCILLATIONS
}

\author{
Clara Ionescu, Robin De Keyser \\ Ghent University, EeSA Department of Electrical energy, Systems \& Automation \\ Technologiepark 913, B-9052, Gent, Belgium \\ Tel.32-9-2645608, Fax.32-9-2645603,E-mail: \{clara,rdk\}@autoctrl.UGent.be
}

\begin{abstract}
The objective of this paper is to present recent results within an investigation on human respiratory mechanics. The overall aim is to provide a fast method for identification of airway mechanics, in order to assist the medical staff in obtaining a diagnosis of the patient. Some ideas for a novel detection of respiratory mechanics parameters are presented, as well as preliminary experimental results, indicating the future potential of the method. Copyright (C) 2005 IFAC
\end{abstract}

Keywords: signal processing, filter design, amplitude modulation, frequency dependent characteristics, forced oscillation, sinusoidal signals

\section{INTRODUCTION}

Respiratory mechanics have been intensively studied in the last decades and the results are still used nowadays. Although many scientists are working on signal processing methods, the potential of those methods in the field of respiratory mechanics is not yet fully explored.

Current research related to respiratory mechanics tends to provide information on the interaction between other systems and the respiratory system, especially the cardiovascular system, since it involves gas exchange and perfusion phenomena (Liu, et al., 1998). Another trend is to obtain a model of the normal human lung, for analysis under different stimuli (Liu, et al., 1998; Mañanas, et al., 2002).

One of the popular non-invasive lung function tests (Pride, 1996) is the Forced Oscillation Technique (FOT). The technique is mainly used in characterization of the respiratory function, assessment of bronchial challenge, mechanical ventilation and sleep studies (Van Noord, 1990; Navajas and Farré, 1999). FOT has also been successfully used in investigation of the respiratory oscillation mechanics in infants and preschool children (Marchal and Loos, 1997) and in obtaining a parametric model of the (nonlinear) human lung mechanics (Ionescu and De Keyser, 2003).

In this contribution, FOT is applied to a specific group of subjects and the role of signal processing (Orfanidis, 1996) is enhanced and directed towards analysis with respect to diagnosis of the human respiratory system. A FOT-phenomenon which occurs during breathing presents special interest and a brief description of the required signal processing is given in (Ionescu and De Keyser, 2004). Another assessed aspect is the low frequency range used in the analysis: $1-9 \mathrm{~Hz}$, close to the breathing frequency $(\approx 0.3 \mathrm{~Hz})$. The normal breathing of the subject has to be regarded as 'noise' because undesired interference with the (low frequency) excitation signal is possible.

The paper is organized as follows: the used apparatus and the principle of forced oscillation are briefly described in Section 2. Signal processing of the timesignals achieved from the patient is detailed in the $3^{\text {rd }}$ Section. In Section 4, similar tests have been made with another 2 subjects in order to emphasize the potential of the method for evaluating respiratory mechanics and a brief discussion of the results is made in Section 5. Conclusions and future developments are given in a final Section. 


\section{APPARATUS AND LUNG FUNCTION TEST}

The mechanical properties of the respiratory system may be obtained by applying the Forced Oscillation Technique (FOT), measuring thus the airflow $\mathrm{Q}(\mathrm{t})$ and trans-respiratory pressure $\mathrm{P}(\mathrm{t})$. Its simplicity and non-invasive technique makes it ideal for routine evaluation of the respiratory function (Van Noord, 1990).

The conventional FOT set-up illustrated in Figure 1 is based on superimposing a low-amplitude pressure oscillation at the mouth while the patient is breathing spontaneously. The following notations apply: $\mu \mathrm{C}$ microcomputer; LS - loudspeaker; BT - bias-tube; BF - bias-flow; PN - pneumotachograph; bf biological filter; A - amplifier; PT - pressure transducer; $\mathrm{Q}$ - flow and $\mathrm{P}$ - pressure.

The oscillation pressure is generated by a loudspeaker connected to a chamber, driven by a power amplifier fed with the oscillating signal generated by a computer. The movement of the loudspeaker cone generates a pressure oscillation inside the chamber, which is applied to the patient's respiratory system by means of a tube connecting the loudspeaker chamber and the biological filter (mouthpiece). As the patient breathes spontaneously through a bias tube a constant bias flow avoids rebreathing of used air. It is advisory that during the measurements, the patient should wear a nose clip and keep the cheeks firmly supported.

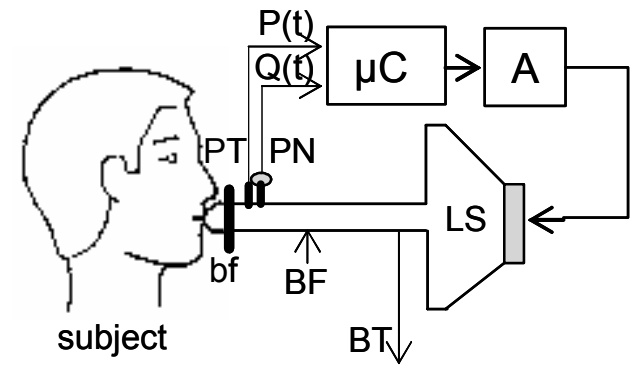

Fig. 1: Conventional FOT set-up

Pressure and flow are measured at the mouthpiece, respectively by means of 1) a pressure transducer (PT) and 2) a pneumotachograph (PN) plus a differential pressure transducer.

These signals are then sampled and stored in a microcomputer and digitally processed as described further in the next section. Before starting the measurements, the frequency response of the transducers and of the pneumotachograph are calibrated to a constant value (Van de Woestijne, et al., 1994).

The signal superimposed on the breathing of the patient is a sinusoidal oscillation since it provides a direct interpretation of the mechanical load of the respiratory system and has the advantage of providing the highest signal-to-noise ratio (SNR). In this paper, the breathing of the patient must be considered as noise.

\section{SIGNAL PROCESSING}

\subsection{Data Gathering with FOT test}

In a preliminary test, one single sinusoid at a time has been considered to be the excitation signal applied to the respiratory system. Three patients have been used to gather the respiratory pressure $\mathrm{P}(\mathrm{t})$ and flow $\mathrm{Q}(\mathrm{t})$ and their characteristics are listed in the table below (COPD - Chronic Obstructive Pulmonary Disease). The preliminary diagnosis was made based on the standard $4-48 \mathrm{~Hz}$ FOT multisine test (Navajas and Farré, 1999).

Table 1: Parameters of the 3 volunteer subjects.

\begin{tabular}{llll}
$\#$ & Age & Gender & Diagnosis \\
\hline A & 67 & M & suspected \\
B & 73 & M & healthy \\
C & 92 & M & asthma+COPD \\
\hline
\end{tabular}

The experiments consisted of a 3,5 and $7 \mathrm{~Hz}$ sinusoid excitation signal superimposed on the breathing of the patient (sampling frequency: $1000 \mathrm{~Hz}$ ). The resulting trans-respiratory pressure $\mathrm{P}(\mathrm{t})$ and air-flow $\mathrm{Q}(\mathrm{t})$ are depicted in Figure 2 (for $5 \mathrm{~Hz}$ ). Since the best SNR is provided by the pressure measurement $\mathrm{P}(\mathrm{t})$, this signal will be further analysed and processed. Figure 2 presents six breathing cycles of approximately 4 seconds per cycle, which correspond to a breathing frequency of about $0.24 \mathrm{~Hz}$.

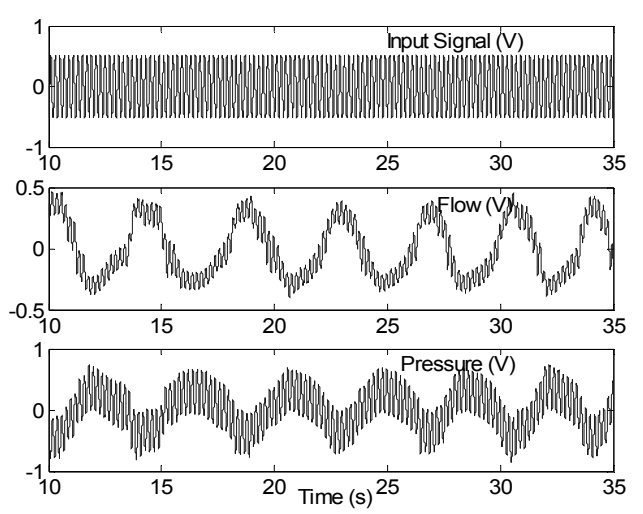

Fig. 2: Input and Output Signals for Subject B (5Hz)

\subsection{Separation of the Signals by Filtering}

The output signals $\mathrm{P}(\mathrm{t})$ and $\mathrm{Q}(\mathrm{t})$ result from the effect of two components: the breathing signal, with a frequency around $0.24 \mathrm{~Hz}$, and the effect of the $5 \mathrm{~Hz}$ test sinusoid sent from the FOT apparatus via the mouthpiece to the patient. Since the breathing and the input signals have frequencies which do not overlap (Fig. 5a) then it is possible to separate them with a $6^{\text {th }}$ order low-pass Chebyshev filter, with the magnitude response characteristic depicted by Figure 3. As a result after low-pass filtering, the breathing signal is obtained (Fig. 4b). By subtracting the breathing signal from the pressure $\mathrm{P}(\mathrm{t})$, the signal in Figure $4 \mathrm{a}$ is obtained. 


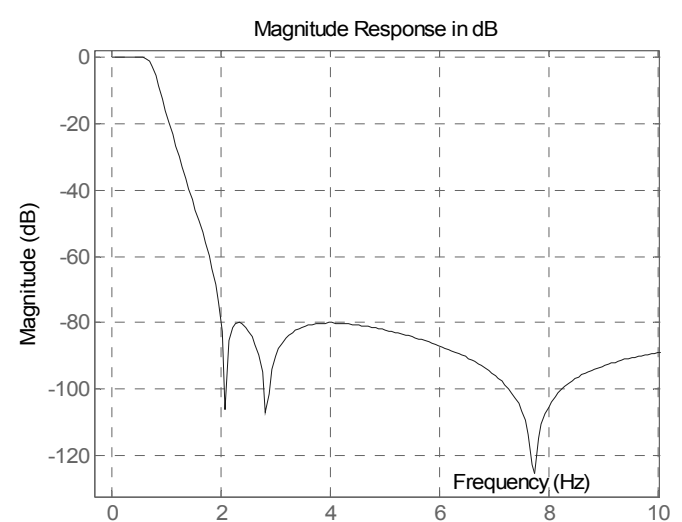

Fig. 3: Chebyshev low-pass filter characteristic
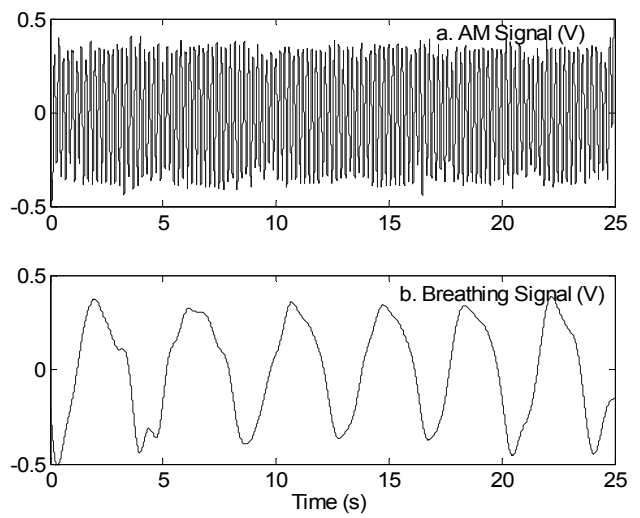

Fig. 4: Time-signals after low-pass filtering
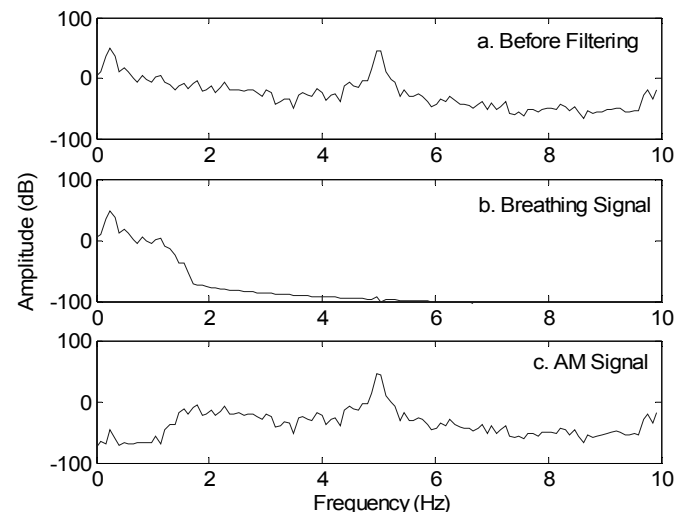

Fig. 5: Spectrum for: $a$. the trans-respiratory pressure signal $\mathrm{P}(\mathrm{t}) ; b$. the breathing signal; $c$. the remaining (AM) signal

From the spectrum depicted in Figure 5, it is observed that harmonics of the input signal $(5 \mathrm{~Hz})$ are introduced $(10 \mathrm{~Hz})$. This is a property of nonlinear systems, assessing the nonlinearity of the human respiratory system. On the other hand, when separating the breathing signal from the pressure signal gathered from subject $\mathrm{B}$, one would expect to remain with a sinusoidal signal of $5 \mathrm{~Hz}$ (of different amplitude and eventually shifted in phase) similar to the input signal of $5 \mathrm{~Hz}$. The remaining signal (Fig. 4a) is indeed a $5 \mathrm{~Hz}$ sinusoid, but it seems to be amplitude modulated (AM) with a signal highly correlated to the breathing frequency of the subject.

Assuming that the modulating signal has the breathing frequency $\left(\omega_{b}\right)$ then the AM signal can be written in the form: where:

$$
s_{m}(t)=\left(A+b \sin \omega_{b} t\right) \sin \left(\omega_{i n} t+\alpha\right)
$$

$\checkmark A$ is a positive constant (the DC component of the modulating signal);

$\checkmark \quad \omega_{i n}=2 \pi f_{\text {in }}$ with $f_{\text {in }}$ the 'carrier' frequency, in this case $5 \mathrm{~Hz}$; and $\alpha$ its corresponding phase shift;

$\checkmark \quad \omega_{b}=2 \pi f_{b}$ with $f_{b}$ the breathing frequency, about $0.24 \mathrm{~Hz}$;

Denoting $s(t)=b \sin \omega_{b} t$, then we can observe from Figure 4 that $|s(t)|<A$ for all $t$, thus $[s(t)+A]$ never goes negative.

The modulation effect might be related to nonlinear properties of the respiratory system and could vary from patient to patient. As a consequence, the modulation-intensity could be a measure able to distinguish variations, being thus a supplementary parameter in the diagnosis procedure.

\subsection{Demodulation}

In Figure 5c it can be observed that the frequency component is not limited to the input signal frequency only $(5 \mathrm{~Hz})$, but contains also harmonics $(10 \mathrm{~Hz})$. Another observation is that it contains sidelobes around $5 \mathrm{~Hz}$ and $10 \mathrm{~Hz}$ frequencies. This is a further indication that the signal is amplitude modulated, and the respective peaks can be foreseen from (1) if trigonometric identities are applied:

$$
s_{m}(t)=A \sin \left(\omega_{i n} t+\alpha\right)+b \sin \omega_{b} t \sin \left(\omega_{i n} t+\alpha\right)
$$

with further processing leading to:

$$
\begin{aligned}
& s_{m}(t)=A \sin \left(\omega_{i n} t+\alpha\right)+ \\
& \frac{b}{2}\left[\cos \left(\left(\omega_{i n}-\omega_{b}\right) t+\alpha\right)-\cos \left(\left(\omega_{i n}+\omega_{b}\right) t+\alpha\right)\right]
\end{aligned}
$$

thus creating the side-lobes around $5 \mathrm{~Hz}$ frequency.

One of the classical demodulation methods is multiplying (1) with a sine - or cosine - function at the carrier frequency $(5 \mathrm{~Hz}$ in this case). However, the successful result is obtained only if the signals are not shifted in time (see Appendix). In this FOT application this condition is hard to achieve. Another way to achieve demodulation is to investigate the effect of squaring the signal (Roden, 1991).

$$
\begin{aligned}
s_{m}^{2}(t) & =\left[\left(A+b \sin \omega_{b} t\right) \sin \left(\omega_{i n} t+\alpha\right)\right]^{2} \\
& =\left[A+b \sin \omega_{b} t\right]^{2} \sin ^{2}\left(\omega_{i n} t+\alpha\right) \\
& =\left[A+b \sin \omega_{b} t\right]^{2} \frac{1-\cos \left(2 \omega_{i n} t+\gamma\right)}{2}
\end{aligned}
$$

$$
=\frac{\left[A+b \sin \omega_{b} t\right]^{2}}{2}-\frac{\left[A+b \sin \omega_{b} t\right]^{2} \cos \left(2 \omega_{i n} t+\gamma\right)}{2}
$$


From (7) the signal $0.5\left[A+b \sin \omega_{b} t\right]^{2}$ can be extracted by low-pass filtering $\left(6^{\text {th }}\right.$ order Butterworth filter) as frequencies do not overlap (i.e. left-hand term contains the $0, \omega_{b}$ and $2 \omega_{b}$ frequencies, while the right-hand term contains the $2 \omega_{i n}, 2 \omega_{i n} \pm \omega_{b}$ and $2 \omega_{\text {in }} \pm 2 \omega_{b}$ frequencies).

It is possible to take the positive square root of the $1^{\text {st }}$ term, obtained after LPF, in order to get $0.707|s(t)+A|$. Taking the magnitude $|\ldots|$ of a signal represents a severe form of distortion, but it was already stated that $A$ is larger than the amplitude of $s(t)$ so that $s(t)+A$ never goes negative. In that case, the magnitude $|s(t)+A|$ is equal to $s(t)+A$ and demodulation is accomplished. The results can be observed in Figure 6.
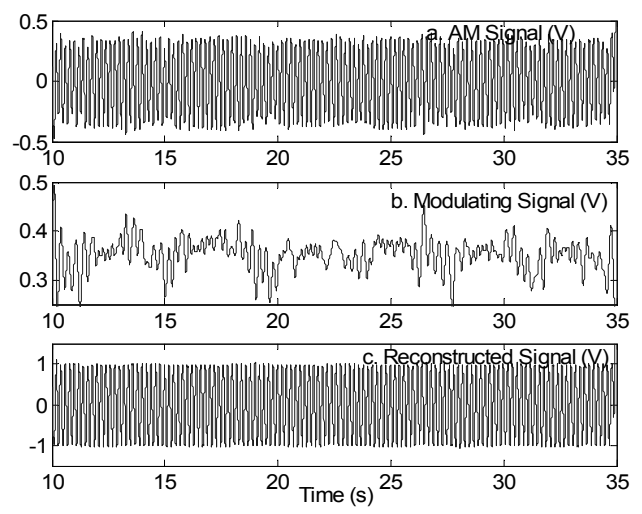

Fig. 6: Time signals for: $a$. the amplitude modulated signal; $b$. the extracted modulating signal; $c$. the reconstructed signal

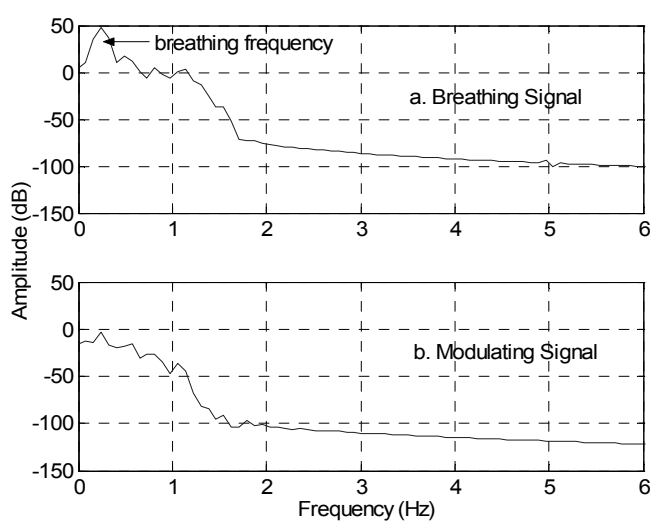

Fig. 7: Spectrum for: $a$. the breathing signal; $b$. the modulating signal

In Section 3.2 it has been stated that the modulation could be related to the breathing frequency of the subject. Figure 7 depicts the spectrum of the breathing signal (as in Figure $4 \mathrm{~b}$ ) and the modulating signal (as in Figure 6b).

In the spectrum of the modulating signal (Fig.7b) peaks close to the breathing frequency (and its double and multiple frequency) component can be observed. It can be stated that the modulating signal (Fig. 6b) contains a frequency related to the breathing (i.e. signal is periodic at time $10 \mathrm{~s}, 15 \mathrm{~s}$,
$20 \mathrm{~s}, \ldots)$. This observation supports the assumption that the $5 \mathrm{~Hz}$ signal is AM by a signal correlated with the natural breathing of the subject, and could vary from one patient to another.

\section{ASSESSMENT OF AIRWAY MECHANICS}

In the previous section, the $5 \mathrm{~Hz}$ pressure signal $\mathrm{P}(\mathrm{t})$ from patient $\mathrm{B}$ has been decomposed in the breathing signal and the AM signal. Further on, demodulation has been accomplished and the initial signal has been reconstructed. The result of the demodulation is the modulating signal (Fig. 6b). If one calculates its mean value $\mu$ and its standard deviation $\sigma$ for each frequency point in the $1-9 \mathrm{~Hz}$ interval, then a frequency-dependent characteristic is obtained. Notice that in this faze, the experiments are performed consecutively for each frequency. The 3, 5 and $7 \mathrm{~Hz}$ frequencies have been chosen from the 1$9 \mathrm{~Hz}$ interval. Having the measurements for these frequencies it is possible to get an overview upon the allure of the frequency-dependent characteristic.

Repeating the signal processing described in section 3 for the other two frequencies ( 3 and $7 \mathrm{~Hz}$ ) for the same subject $B$ then the continuous line characteristic in Figure 8 is achieved. Applying the entire procedure to the other 2 volunteers $\mathrm{A}$ and $\mathrm{C}$, the dashed and respectively dotted characteristics in Figure 8 are obtained.
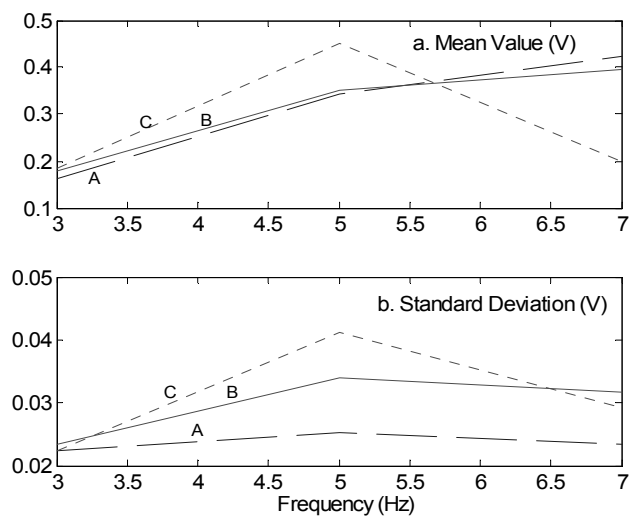

Fig. 8: Mean Values (a) and Standard Deviation (b) for Subject A (dashed line), Subject B (continuous line) and Subject C (dotted line)

The fact that the standard deviation of the modulating signal remains approximately constant for patients A and $\mathrm{B}$ after $5 \mathrm{~Hz}$ frequency, indicates the fact that the breathing frequency is not so strongly correlated to the excitation signal when the frequency increases. For the same patients, an increase in the mean value with frequency indicates the fact that a $7 \mathrm{~Hz}$ sinusoid has a stronger impact on the respiratory system than a $3 \mathrm{~Hz}$ sinusoid in the excitation signal. The pathologic case $\mathrm{C}$ (dotted line - Asthma \& COPD) is distinguished from the other 2 subjects, a difference in quantitative terms being observed.

Consider the following measure: the modulation index $(\mathrm{MI})$ in the recorded signal $\mathrm{P}(\mathrm{t})$ is defined as 
the ratio between the standard deviation of the modulating signal over its mean value:

$$
\mathrm{MI}=\frac{\sigma}{\mu}
$$

Calculating the MI coefficient for each frequency and for each of the three subjects, then the characteristics in Figure 9 are obtained. Supplementary confidence intervals based on 5 measurements of a healthy subject are given in Figure 10.

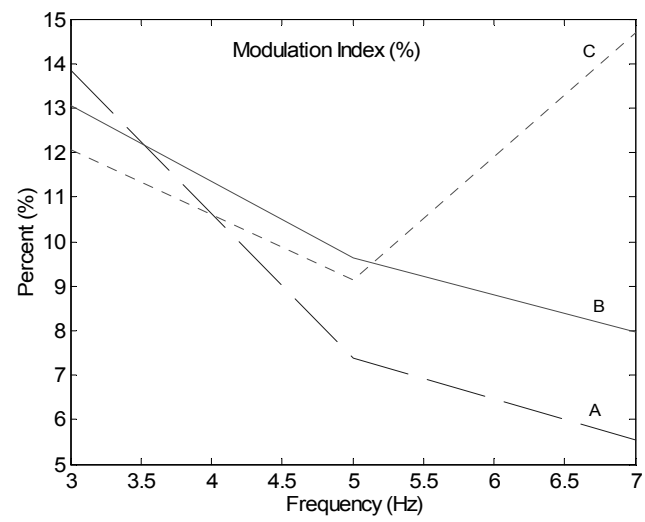

Fig. 9: Modulation index for subject A (dashed line), subject B (continuous line) and subject C (dotted line)

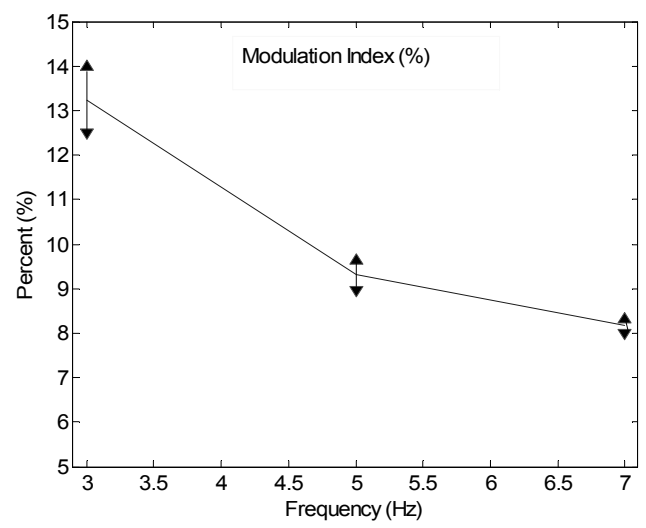

Fig. 10: Modulation index (mean \pm SD)

\section{DISCUSSION}

Airflow obstruction and narrowing of the airways can be caused by different pathological conditions (Van Noord, 1990). Asthma is characterized by variable airway narrowing due to bronchial muscle contraction, edema and infiltration of the bronchial mucosa. In COPD the morphological basis of the largely irreversible airflow limitation are varying combinations of inflammatory and fibrotic narrowing of the peripheral airways and loss of lung elastic recoil.

Although asthma and COPD (i.e. chronic bronchitis) are defined as distinct and separate disorders, many patients show evidence of more than one entity and the classification of these overlap patients is often difficult. Subject $\mathrm{C}$ is one of these overlapping cases.
After decades of tests and improvements of the FOT it has been assessed that it is one of the easiest to perform lung function tests (Pride, 1996). It has been also concluded that FOT in its own is not able to distinguish the various obstructive and restrictive disorders of the respiratory system on the basis of a specific pattern of frequency-dependent curves (Van Noord, 1990).

Despite previous assessment of FOT as an additional information method and not as a clear diagnosis method, the present contribution aims to detect novel use of FOT in assessment of airway mechanics parameters. The (nonlinear) effects of the human respiratory system in response to an excitation signal, i.e. introducing harmonics and amplitude modulation, are novel within respiratory mechanics investigation.

FOT has also the advantage that the frequency band may be modified depending on the pathophysiological aim of the measurement (Navajas and Farré, 1999). For instance, if the interest is focused on studying respiratory tissue properties, low frequency data would be more sensitive (since it is closer to the breathing frequency of the patient).

Although the frequency-dependent curves of the subjects overlap, there can be considered quantitative and allure differences between the three characteristics.

The method presented in this paper can lead to a diagnosis method under certain conditions. The most important condition is that the SNR has to be improved. The measurement should be done at allfrequencies from $1-9 \mathrm{~Hz}$ in a fast manner (i.e. onestep multi-sine test signal). Consequently, the filtering has to be adapted; the use of notch filters could hold a satisfactory solution.

\section{CONCLUSIONS}

In this contribution, a preliminary investigation has been presented as well as results considering a potentially novel method of diagnosis for the human respiratory system. The experiments are based on the principles of the well-known Forced Oscillation Technique (FOT).

FOT is - despite the relative simplicity considering its use by medical staff - a promising method to investigate respiratory mechanics, thus being suited for use in clinical medicine. In this application, the oscillatory pressure has been superimposed on the spontaneous (normal) breathing of the patient.

The use of a single-frequency sinusoid as a test input has proved to be successful for observing interesting phenomena. The results obtained so far provide new insights into the complexity of the human respiratory system. Showing nonlinearities and mechanic properties, they are a strong basis to motivate further research and investigation.

One of the further ideas would be to optimize a multi-sine input signal, in order to obtain the 
patient's characteristic in a one-step experiment, i.e. over a full test-range of (low) frequencies. Also, a larger number of subjects could help providing a trend in the frequency dependent characteristics under different pathologic conditions.

\section{ACKNOWLEDGEMENTS}

The authors appreciate the support given by $M r$. Rudy Frigne from CHESSmt company Belgium, during this investigation.

\section{REFERENCES}

Ionescu C., R. De Keyser (2003). A novel parametric model for the human respiratory system. In: Proc. of the $14^{\text {th }}$ IASTED International Conference on Modeling \& Simulation, (Hamza M.H. (Ed)), 246-251. Acta press, Anaheim.

Ionescu C., R. De Keyser (2004). Towards a novel non-invasive diagnosis method for the human respiratory system. In: CD-Proc. of the IEEE $X$ Medit. Conf. on Medical and Biological Engineering, MEDICON and Health Telematics, (ISSN 1727-1983), 4p, IFMBEAIIMB, Naples

Liu C.H., S.C. Niranjan, J.W; Clark, jr., K.Y. San, J.B. Zwischenberger, A. Bidani (1998). Airway mechanics, gas exchange and blood flow in a nonlinear model of the normal human lung. Journal of Applied Physiology, 77, 1447-1470.

Mañanas M.A., C. Navarro, S. Romero, R. Griño, R. Rabinovich, S. Benito, P. Caminal (2002). Control system response of different respiratory models under ventilatory stimuli and pathologies. IFAC Proc. of the $15^{\text {th }}$ Triennial World Congress. Barcelona, Spain, 6p

Marchal F., N. Loos (1997). Respiratory oscillation mechanics in infants and preschool children. European Respiratory Monograph, 5, 58-87

Navajas D., R. Farré (1999). Oscillation mechanics. European Respiratory Monograph, 12, 112-140

Orfanidis S. (1996). Introduction to Signal Processing. Prentice Hall, NJ.

Pride N.B. (1996). Lung function tests physiological principles and clinical applications, (J.M.B. Hughes, N.B. Pride. (Ed)), 3-72. W.B. Saunders, London:

Roden M.S. (1991). Analog and Digital Communication Systems. Chapter 4. Prentice Hall, NJ

Van de Woestijne K.P., K. Desager, E. Duiverman (1994). Recommendations for measurement of respiratory input impedance by means of forced oscillation technique. European Respiratory Review, 4, 235-237

Van Noord J.A. (1990). Oscillation mechanics of the respiratory system: Clinical applications and modeling. Katholieke Universiteit, Leuven.

\section{APPENDIX}

Suppose the signal to be demodulated is of this form:

$$
s_{m}(t)=\left(A+b \sin \omega_{b} t\right) \sin \left(\omega_{i n} t+\alpha\right)
$$

then the classical demodulation method is applied by multiplication with a sine function at the carrier frequency:

$$
s_{m}(t)=\left(A+b \sin \omega_{b} t\right) \sin \left(\omega_{i n} t+\alpha\right) \cdot \sin \left(\omega_{\text {in }} t+\beta\right)(2 \mathrm{a})
$$

where $\alpha$ and $\beta$ denotes the phase shift in the signal.

Introducing trigonometric identities, (2a) becomes:

$$
\begin{aligned}
& \sin \left(\omega_{i n} t+\beta\right)\left\{A \sin \left(\omega_{i n} t+\alpha\right)+\right. \\
& \left.\frac{b}{2}\left[\cos \left(\left(\omega_{i n}-\omega_{b}\right) t+\alpha\right)-\cos \left(\left(\omega_{i n}+\omega_{b}\right) t+\alpha\right)\right]\right\}
\end{aligned}
$$

and if brackets are omitted, then remains:

$$
\begin{aligned}
& A \sin \left(\omega_{i n} t+\alpha\right) \sin \left(\omega_{i n} t+\beta\right)+ \\
& +\frac{b}{2} \cos \left(\left(\omega_{i n}-\omega_{b}\right) t+\alpha\right) \sin \left(\omega_{i n} t+\beta\right)- \\
& -\frac{b}{2} \cos \left(\left(\omega_{i n}+\omega_{b}\right) t+\alpha\right) \sin \left(\omega_{i n} t+\beta\right)
\end{aligned}
$$

Evoking trigonometric identities once again, (4a) can be detailed to:

$$
\begin{aligned}
& \frac{A}{2} \cos \left(\left(\omega_{i n}-\omega_{i n}\right) t+\alpha-\beta\right)- \\
& -\frac{A}{2} \cos \left(\left(\omega_{i n}+\omega_{i n}\right) t+\alpha+\beta\right)+ \\
& +\frac{b}{4} \sin \left(\left(\omega_{i n}+\omega_{i n}-\omega_{b}\right) t+\alpha+\beta\right)- \\
& -\frac{b}{4} \sin \left(\left(\omega_{i n}-\omega_{i n}-\omega_{b}\right) t+\alpha-\beta\right)- \\
& -\frac{b}{4} \sin \left(\left(\omega_{i n}+\omega_{i n}+\omega_{b}\right) t+\alpha+\beta\right)+ \\
& +\frac{b}{4} \sin \left(\left(\omega_{i n}-\omega_{i n}+\omega_{b}\right) t+\alpha-\beta\right)
\end{aligned}
$$

and if defining: $\alpha+\beta=\delta, \alpha-\beta=\gamma$ then (5a) becomes:

$$
\begin{aligned}
& \frac{A}{2} \cos (\gamma)-\frac{A}{2} \cos \left(2 \omega_{i n} t+\delta\right)+ \\
& +\frac{b}{4} \sin \left(\left(2 \omega_{i n}-\omega_{b}\right) t+\delta\right)-\frac{b}{4} \sin \left(-\omega_{b} t+\gamma\right)- \\
& -\frac{b}{4} \sin \left(\left(2 \omega_{i n}+\omega_{b}\right) t+\delta\right)+\frac{b}{4} \sin \left(\omega_{b} t+\gamma\right)
\end{aligned}
$$

Low pass filtering leads to

$$
\frac{A}{2} \cos \gamma+\frac{b}{4} \sin \left(\omega_{b} t+\gamma\right)-\frac{b}{4} \sin \left(-\omega_{b} t+\gamma\right)
$$

which gives the modulating signal $\left(A+b \sin \omega_{b} t\right)$ only if $\gamma=0$. 